\title{
Vehicle routing Problem and its Algorithm
}

\author{
Juping Peng ${ }^{1, a} \quad$ Yueguang $\mathrm{Li}^{2, \mathrm{~b}}$ \\ ${ }^{1}$ Gansu Normal University for Nationalities, Hezuo, gansu, 747000, \\ China \\ ${ }^{2}$ Gansu Normal University for Nationalities, Hezuo, gansu, 747000, \\ China \\ ${ }^{a}$ Yueguangli7@163.com, ${ }^{b}$ Yueguangli7@sohu.com
}

\begin{abstract}
The vehicle routing problem, it is a many factor of the global optimization problem. In this paper, according to the characteristics of school bus problem, a Plant Growth Simulation Algorithm(PGSA) was put forward, and it was used to solve the vehicle routing problem, the algorithm was experimented and the experimental results show that the new algorithm has reliable convergence, high convergence rate and solution precision.

Keywords: Plant Growth Simulation Algorithm(PGSA);Vehicle routing problem; Intelligent algorithm
\end{abstract}

\section{Introduction}

In recent years, the vehicle routing problem was widely concerned, from the safety, management mode, route planning and city traffic pressure, all aspects need management and city planners proposed solutions. At home and abroad, the academic research of the route selection and the bus vehicle scheduling problem are still difficult, management and decision analysis need involve the massive spatial data and attribute data, an efficient and feasible algorithm can use the least cost to improve school bus service quality.

This kind of problem can be described as follows ${ }^{[1]}$ : Given a directed graph $\mathrm{G}=(\mathrm{V}, \mathrm{E})$, where $\mathrm{V}$ is the school bus stop collection, $\mathrm{E}$ is the set of edges, it is weighted edges, and each side has a service demand $\mathrm{qij} \geq 0$ ( $\mathrm{i}, \mathrm{j}$ for two adjacent a stop, and $i, j \in V$ ), how to find a loop, all sides of demand of the loop are satisfied and the total service costs least. Intelligent optimization algorithms, such as 
dynamic programming algorithm, nonlinear programming ${ }^{[2]}$; heuristic algorithm: SFC ${ }^{[3]}$, tabu search algorithm ${ }^{[4]}$, genetic algorithm ${ }^{[5]}$ and a variety of hybrid algorithm etc ${ }^{[6]}$. The intelligent algorithms have been paid more and more attention, they are used to solve combinatorial optimization ${ }^{[7]}$.

In order to solve the above problems, this paper puts forward a Plant Growth Simulation Algorithm(PGSA) to solve vehicle routing problem, provide decision support for the practice of route planning.

\section{The mathematical model of vehicle routing problem}

Mathematical models are as follows:

$\sum_{\text {Minmi }} \sum_{I \in V} \sum_{j \in V} X_{i j k}\left(C_{i j}+C_{i j}^{\prime}\right)+F$

$\sum_{\text {s.t }} \sum_{i \in V} \rho_{i} X_{i j k} \leq Q_{k} \quad \forall k \in S$

$\sum_{i \in V} X_{i p k}-\sum_{j \in V} X_{p j k}=0$

$\sum_{k \in S} \sum_{j \in V} \sum_{i \in V} X_{i j k}-Z_{i} \geq 0$

$R_{i}-R_{j}+V * \sum_{k \in S} X_{i j k} \leq V-1 \quad \forall i, j \in V, i \neq j$

$X_{i j k} \in\{0,1\} \quad \forall i, j \in V, \forall k \in S, i \neq j$

$Z_{i} \in\{0,1\} \quad \forall i \in V$ 
Among them: $C_{i j}$ Express the average cost of transportation stop $i$ to $j$, when the edge has Services; $C_{i j}^{\prime}$ express the average cost of transportation stop $i$ to $j$, when the edge hasn't Services; $X_{i j k}$ is the decision variables of service edge, when the vehicle $\mathrm{K}$ across stop $i$ to stop $j$ is 1 , or it is 0 ; $\mathrm{F}$ is average fixed of driver; $\rho_{i}$ is the service quantity of stop $i$; $Q_{k}$ is the maximum capacity of vehicle $\mathrm{K} ; Z_{i}$ indicating whether or not stop at stop $i$, when stop is 1 , or it is 0 ; $V$ is the set of all docked points; $\mathrm{S}$ is the set of all vehicles.

Equation (1) meets the minimum required services; Equation (2) is transport capacity constraints, it meets each bus does not exceed its capacity in a line running; Equation (3) guarantees the route successive; Equation (4) ensures has at least one customer at each docking point ; Equation (5) ensures that it has not internal closed loop on the vehicle routes; Equation (6) and Equation (7) satisfying the integer constraint.

\section{The plant growth simulation algorithm}

Plant growth simulation algorithm(PGSA) is an evolutionary computation technique through simulating the growth processes in plants, which is based on the principles of plant phototropism growth ${ }^{[8]}$. The algorithm has a good prospect in the application due to requiring a simple on the parameters. It has gradually been applied in the field of engineering by many scholars ${ }^{[9]}$.

Plants can be viewed as a system, which composes of a large number of branches and nodes. It must be as soon as possible to strive for breeding more branches and leaves for earning more surface areas, which can obtain the greatest possible sunlight. The form of grammar that simulates the plant description analysis and development had been established since linguistics was introduced into the biological, which based on a simple rewrite rules and branching rules, this is called L-system. Formal description of plant growth can be carried out as the following: 
1. The section of the site grow new branches which first emerged in a number of stems is called the growth node.

2. Most of the new branches have grown updated branches, and the process repeated on the old and new branches.

3. Different branches in the tree have similarities with each other, and the entire plant has self-similar structure.

The Branching model of plant growth is improved according to L-system combined with computer graphics and fractal theory, the plant, as the form of plant growth characteristics, can be described as follow: Supposed branches of plant growth occurred in the two-dimensional plane, Each branch growing in units length every time, or rotating a certain angle $\alpha$, Starting from the node of trunk or branches, The growth process were repeated through rewriting the rules of branches of plant growth in the two dimensional plane.

It was proved by biological experiments that a new branch that is able to grow depends on its morphactin concentration when the plant has more than one node. The node which has larger value of morpheme concentration has more growth opportunities than the smaller one, morphactin concentration are not pre-assigned to the nodes, but formed according to their location information which we call that plant showed the feature of plant's Phototropism. After the node has been formed, morphactin concentration will be readjusted according to changing in the environment of a new growth node else.

We could describe the characteristics of plant growth in term of the mathematical view basis on plant growth analysis of the above. Suppose the length of tree trunk is $\mathrm{T}$, the length of branch is $\mathrm{L}$, there are $\mathrm{W}$ growing nodes $S_{T}=\left(S_{T 1}, S_{T 2}, \ldots, S_{T W}\right)$ in the trunk, morphactin concentration of those nodes is $P_{T}=\left(P_{T 1}, P_{T 2}, \ldots, P_{T W}\right) \quad$ respectively. There are $q$ growth nodes $S_{T}=\left(S_{T 1}, S_{T 2}, \ldots, S_{T q}\right)$ in the branch. Morphactin concentration of those nodes is $P_{T}=\left(P_{T 1}, P_{T 2}, \ldots, P q\right)$ respectively. The morphactin concentration which grown in the trunk and branch is calculated as follows: 


$$
P_{T i}=f\left(x_{0}\right)-f\left(S_{T i}\right) / \sum_{i}^{W}\left(f\left(x_{0}\right)-f\left(S_{T i}\right)\right)+\sum_{j}^{q}\left(f\left(x_{0}\right)-f\left(x_{T q}\right)\right)
$$

Where $x_{0}$ represents the root of plant (the initial node), $f(*)$ is the information function of the node in the environment, The smaller value of the function as possible shows the better environment where the node located, it will help to grow new branch.

From formula (8), the value of morphactin concentration in every node depends on relative position of the relative to the initial root and the location of the environmental information, the mechanism consistent with the formation of the morphactin concentration in the plant cell. We can deduced from formula (8) as follows:

$$
\sum_{i=1}^{T} \sum_{j=1}^{q}\left(P_{T i}+P_{T j}\right)=1
$$

We can produce random number in the interval $[0,1]$. The random number must fall within one of the state space $\left(P_{1}, P_{2}, \ldots, P_{T+q}\right)$, where the corresponding node will have a prior right to grow new branch. A simple example of morphactin concentration state space displays in the fig. 1.The value of morphactin concentration in all nodes will change after the new branch has grown up. Computation formula of the value of morphactin concentration in other nodes will add the relevant node on the new branch, and remove the grown node, which basis on formula (8). The process will be repeated until there is no new branches growing.

\section{The analysis of simulation}

Data from Lanzhou City (Lanzhou University Of Technology lies in Lanzhou City), running regional cooperation for the old campus to the new campus, docked points for 20 , the passenger volume of the bus is 80 , maximum iterative algebra is 2000 in the algorithm, figure 1 is The map of Lanzhou 
University of Technology, Figure 2 shows the best running route map of Lanzhou University of Technology. From the above simulation data shows: this algorithm is very effective for vehicle routing problem.

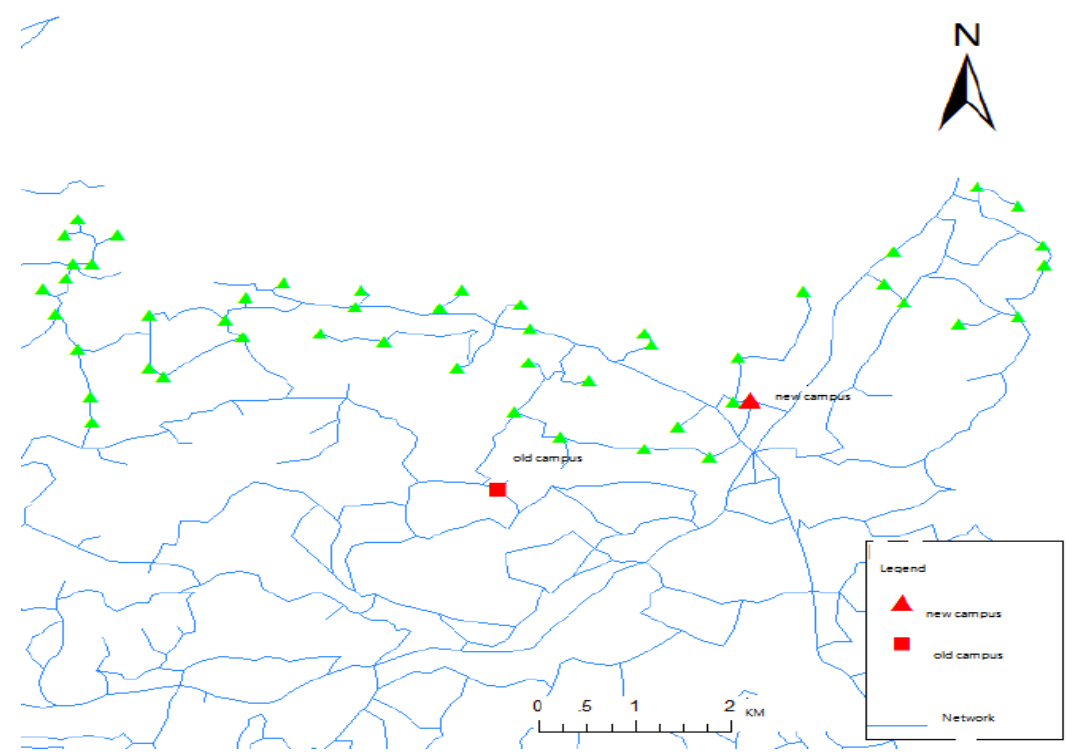

Figure 1 The map of Lanzhou University of Technology

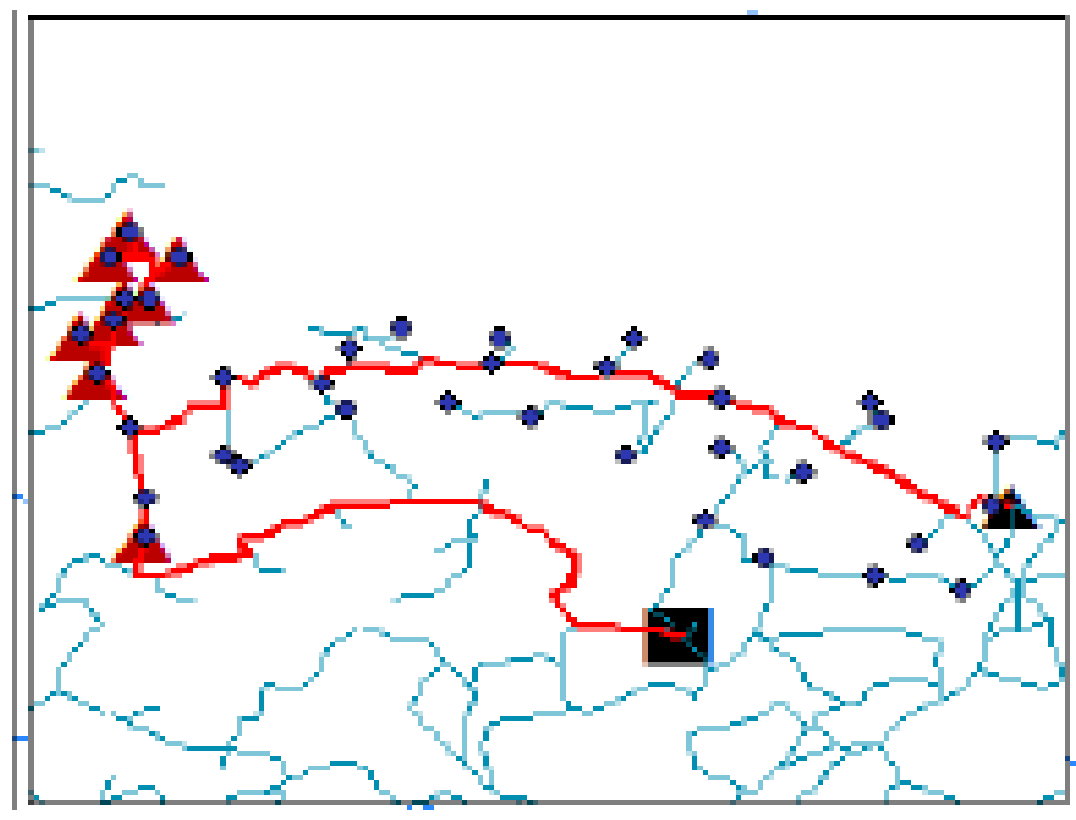

Figure 2 Running route map of Lanzhou University of Technology 


\section{Conclusions}

This paper proposes a plant growth algorithm, the plant growth algorithm is applied to solve vehicle routing problem. Experiments show that the new algorithm has strong global search ability, convergence speed.

\section{References}

[1] Bodin L, Golden B, Assad A, Ball M. Routing and scheduling of vehicles and crews: the state of the art..

[2] Wang Shouyang, Zhao Qiuhong, Xia Guoping. Research on combined location-routing problems in integrated logistics systems[J]. Journal of manegement sciences, 6,69-75(2000).

[3] Chan Y, Carter W B, Burnes M D. A multiple-depot, multiple-vehicle, location-routing problem with stochastically processed demands[J]. Computer \& Operation Research,28,803-826(1999).

[4] Tuzun D, Burke L I. A two-phase tabu search approach to the location routing problem[J]. European Journal of Operational Research, 116,87-99(1999).

[5] Li Minqiang, Kou Jisong, Li Dan. The basic theory of genetic algorithm and its application[M]. Beijing: Science press,1-47(2002).

[6] Wang L. Intelligent Optimization Algorithms Applications [M]. Beijing: Tsinghua University Press,2001.

[7] Reingold. E. M. J. Neivergelt and N. Deo. Combinatorial Algorithms: Theory and practice Prentice-Hall ,Englewood cliffs, NJ(1977).

[8] Li T,Wang C F ,et al.A global optimization bionics algorithm for solving integer Programming plant growth simulation algorithm[J] .Systems Engineering - Theory \& Practice ,2005,25(1) :76 - 85. 
[9] Li T, Wang Z t. Application of plant growth simulation algorithm on solving facility location problem [J]. Systems Engineering - Theory \& Practice, 2008, (12):107-115 